\title{
5-Aminolevulinic acid improves chicken sperm motility
}

\author{
Shin Taniguchi ${ }^{1,2}$, Zhendong Zhu ${ }^{1}$, Mei Matsuzaki ${ }^{3}$, Masaoki Tsudzuki ${ }^{3,4}$, and Teruo Maeda ${ }^{3,4, *}$
}

* Corresponding Author: Teruo Maeda Tel: +81-90-6406-4456,

E-mail: temaeda@hiroshima-u.ac.jp

'Graduate School of Biosphere Science, Hiroshima University, Higashi-Hiroshima 7398528, Japan

2 One Health Business Department, Neopharma Japan Co., Ltd., Fujimi, Chiyodaku, Tokyo 102-0071, Japan ${ }^{3}$ Graduate School of Integrated Sciences for Life, Hiroshima University, Higashi-Hiroshima 739-8528, Japan

${ }^{4}$ Japanese Avian Bioresource Project Research Center, Hiroshima University, Higashi-Hiroshima 739-8528, Japan

ORCID

Shin Taniguchi

https://orcid.org/0000-0003-0358-9327

Zhendong Zhu

https://orcid.org/0000-0001-7002-7119 Mei Matsuzaki

https://orcid.org/0000-0001-8381-754X

Masaoki Tsudzuki

https://orcid.org/0000-0003-0475-7978

Teruo Maeda

https://orcid.org/0000-0001-8049-4386

Submitted Jan 12, 2021; Revised Mar 12, 2021; Accepted Apr 6, 2021
Objective: This study investigated the effects of 5-aminolevulinic acid (5-ALA) on the motility parameters, mitochondrial membrane depolarization, and ATP levels in chicken sperm.

Methods: The pooled semen from Barred Plymouth Rock males was used. In the first experiment, the semen was diluted 4-times with phosphate-buffered saline (PBS (-)) containing various concentrations $(0,0.01,0.05$, and $0.1 \mathrm{mM})$ of 5 -ALA, and then the sperm motility parameters after incubation were evaluated by computer-assisted sperm analysis (CASA). In the second experiment, the semen was diluted 4-times with PBS (-) containing $0.05 \mathrm{mM} 5$-ALA, and then sperm mitochondrial membrane depolarization and ATP levels after $1.5 \mathrm{~h}$ of incubation were analyzed with the MitoPT ${ }^{\circledast}$ JC-1 Assay and ATP Assay kits, respectively. In the third experiment, the semen was removed from the seminal plasma and resuspended with the mediums of PBS (-), PBS (-) supplemented with $\mathrm{CaCl}_{2}$ and $\mathrm{MgCl}_{2}$ $(\mathrm{PBS}(+))+5$-ALA, PBS $(+)+$ caffeine, and PBS $(+)+$ caffeine + 5-ALA. Then, the sperm motility parameters after incubation were evaluated by CASA. In the last experiment, the semen was treated with the mediums of PBS (-), PBS (-) + 5-ALA, 5.7\% glucose, 5.7\% glucose +5-ALA after removing the seminal plasma, and then the sperm motility parameters were evaluated by CASA.

Results: The addition of $0.05 \mathrm{mM}$ 5-ALA significantly increased the chicken sperm motility, progressive motility, linearity, average path velocity, curvilinear velocity, straight-line velocity, and the wobble. The sperm mitochondrial membrane depolarization was also increased by the 5-ALA treatment. The 5-ALA treatment decreased the sperm ATP levels. Both the caffeine treatment and glucose treatment decreased the sperm motility during incubation period.

Conclusion: 5-ALA might increase sperm mitochondrial membrane depolarization to utilize the ATP for enhancing sperm movement.

Keywords: 5-Aminolevulinic Acid; Chicken; Sperm Motility

\section{INTRODUCTION}

5-Aminolevulinic acid (5-ALA) is a delta amino acid that has a carbonyl group at the fourth carbon [1], which naturally presents in plants, animals, algae, and photosynthetic bacteria [2-4]. There are two distinct pathways for 5-ALA to be biosynthesized; from glutamate in plants/bacteria or from the succinyl-coenzyme A and glycine in animals. 5-ALA is regarded as a precursor of heme, which is an essential substance for oxygen metabolism and energy generation in various animals [5]. Morokuma et al [6] reported that 5-ALA significantly enhanced hair growth and cell proliferation. Lee et al [7] demonstrated that the complex of 5-ALA and glycyl-histidyl-lysine peptide was considered as one of the complementary agents for the treatment of male-pattern hair loss. 5-ALA also has been reported to boost the immune system. Sato et al [8] reported that 5-ALA was used as an immunomodulator to stimulate $\mathrm{T}$ cells via mild oxidative stress in growing broiler chickens, 
thereby improving the growth performance. Moreover, when RAW264.7 cells were treated with a lipopolysaccharide stimulator, treatment with exogenous 5-ALA induced heme oxygenase- 1 to downregulate the expression levels of nitric oxide and proinflammatory cytokines (tumor necrosis factor- $\alpha$, cyclooxygenase 2 , interleukin- $1 \beta$, and interleukin-6), indicating that 5-ALA is an anti-inflammatory agent [9]. In recent years, 5-ALA has also been utilized in photodynamic therapy to diagnose brain tumors and determine the area of resection [10,11]. In addition, 5-ALA has been reported to be an important nutrient for plant growth $[12,13]$. Several studies have shown that 5-ALA can improve the salt tolerance of cotton seedlings [14] and cold resistance in rice seedlings [15]. However, to the best of our knowledge, there are still no reports on the effects of 5-ALA on animal reproduction systems. Whether 5-ALA is beneficial for improving the sperm quality remains unclear. Therefore, in the present study, we aimed to examine the effects of 5-ALA on the sperm motility parameters, mitochondrial membrane depolarization, and ATP levels in the chicken.

As the sperm motility patterns could be enhanced by some chemicals, such as caffeine and extracellular $\mathrm{Ca}^{2+}[16]$, we compared the role of caffeine, extracellular $\mathrm{Ca}^{2+}$ and 5-ALA on chicken sperm motility patterns to evaluate whether the mechanism of 5-ALA chemical enhancing sperm motility is similar to that mechanism in caffeine and extracellular $\mathrm{Ca}^{2+}$ or not. Moreover, to investigate whether 5-ALA improve chicken sperm motility by enhancing ATP production via glycolysis pathway or not, we compared the roles of 5-ALA in phosphate-buffered saline (PBS) (-) medium and the 5.7\% glucose medium.

\section{MATERIALS AND METHODS}

\section{Animals}

Five Barred Plymouth Rock males (12 months old) were used. The males were individually caged and maintained in environmentally controlled houses on a $14 \mathrm{~h}$ light: $10 \mathrm{~h}$ dark photoperiod. The feed and water were provided ad libitum. All of the animals used in the present study were handled in accordance with the regulations of the Animal Experiment Committee of Hiroshima University for animal experiments (approval number: C17-21).

\section{Semen collection}

Semen samples were collected using the abdominal massage method of Burrows and Quinn [17]. Semen from five roosters was pooled to avoid the effects of individual males on the sperm motility.

\section{Experimental design}

In the first experiment (Experiment 1), the fresh chicken se- men was diluted with PBS (-); v:v=1:3) containing various concentrations of 5-ALA $(0,0.01,0.05$, and $0.1 \mathrm{mM})$. The treated diluted semen was incubated at $37^{\circ} \mathrm{C}$ for $2 \mathrm{~h}$. The sperm motility was evaluated every $30 \mathrm{~min}$ by computerassisted sperm analysis (CASA).

To investigate how 5-ALA increase the sperm motility, we measured mitochondrial membrane depolarization and ATP levels of the sperm in the presence or absence of $0.05 \mathrm{mM}$ 5-ALA after $1.5 \mathrm{~h}$ of incubation (Experiment 2).

In Experiment 3, semen was removed from the seminal plasma by washing two times and resuspended the sperm with mediums of PBS (-), PBS (-) supplemented with $\mathrm{CaCl}_{2}$ and $\mathrm{MgCl}_{2}(\mathrm{PBS}(+))+5$-ALA, PBS $(+)+$ caffeine and PBS $(+)+$ caffeine +5 -ALA. The sperm motility was evaluated at $15,30,60$, and 90 min points of incubation by CASA. PBS (+) means the PBS medium contained $\mathrm{Ca}^{2+}$ and $\mathrm{Mg}^{2+}$ irons. The concentration of caffeine was $4 \mathrm{mM}$ according to the report by Wishart and Ashizawa [16].

To further evaluate whether the 5-ALA enhanced sperm motility by utilizing ATP or generating ATP, we incubated the other sperm with mediums of PBS (-), PBS (-) + 5-ALA, glucose, glucose + 5-ALA for $1.5 \mathrm{~h}$. The sperm motility was evaluated every $30 \mathrm{~min}$ by CASA (Experiment 4 ). The concertation of glucose was $5.7 \%(\mathrm{~W} / \mathrm{V})$, which is equilibrated with chicken seminal plasma [18].

Evaluation of sperm motility parameters by computerassisted sperm analysis system

According to Zhu et al [19], a total of $10 \mu \mathrm{L}$ sample was placed in a pre-warmed counting chamber after incubation of sperm for different treatments. Sperm tracks ( $0.5 \mathrm{~s}, 45$ frames) were captured at $60 \mathrm{~Hz}$ using a CASA system (HT CASA-Ceros II; Hamilton Thorne, Beverly, MA, USA). More than 200 individual trajectories were recorded in each treatment. The sperm parameters were defined as follows:

Motility (motile): Percentage of motile sperm moving with a path velocity $>12 \mu \mathrm{m} / \mathrm{s}$. Progress motility (progressive motile): Percentage of motile sperm moving with path velocity $45 \mu \mathrm{m} / \mathrm{s}$ and in a straight line for over $80 \%$ of the time. LIN, linearity; VAP, average path velocity; VCL, curvilinear velocity; VSL, straight-line velocity; WOB, wobble.

\section{Evaluation of sperm mitochondrial membrane depolarization}

Sperm mitochondrial membrane depolarization was measured with MitoPT ${ }^{\oplus}$ JC-1 Assay (911, ImmunoChemistry Technologies, llc., Bloomington, MN, USA) according to Zhu et al [20]. Briefly, sperm samples after treatment were incubated with working solution for $5 \mathrm{~min}$ at $37^{\circ} \mathrm{C}$ in the dark. The mitochondrial membrane depolarization was analyzed by flow cytometry using a filter with a bandwidth of 574/26 nm (Attune R NxT Acoustic Focusing Cytometer, 
Invitrogen, Waltham, MA, USA). The mean fluorescence intensity (MFI) of JC-1 orange aggregates were measured. Sperm with high fluorescence intensity indicated sperm with high mitochondrial membrane depolarization. Total of 50,000 sperm events were analyzed.

\section{Measurement of sperm ATP levels}

Intracellular ATP of sperm was measured as described previously by Matsuzaki et al [21]. The ejaculate was incubated with or without $0.05 \mathrm{mM} 5$-ALA for $1.5 \mathrm{~h}$. The sperm was diluted 100-fold with PBS and dissolved in the ATP assay reagent ("Cellno" ATP Assay Reagent Ver.2, TOYO B-Net Co., Ltd., Tokyo, Japan). After incubating at room temperature for $10 \mathrm{~min}$, the chemiluminescence signal was measured using a luminometer (ARVOTMX4, PerkinElmer Japan, Co., Ltd., Yokohama, Japan). Standard curves were prepared from ATP standard using serial dilutions to obtain concentrations of $0.75,1.5$, and $3 \mu \mathrm{M}$. The ATP levels in sperm samples were calculated according to the standard curve and expressed as $\mathrm{pM} / 10^{6}$ of sperm.

\section{Statistical analysis}

All the data were tested for normality and variance homogeneity prior to statistical analysis. The data were transformed by arcsine square root transformation when necessary, and analyzed using JMP 14.2 (SAS Institute Inc., Cary, NC, USA). The data from five replicates for comparison were performed by either two-way analysis of variance followed by TukeyKramer HDS test (motility parameters) or one-way analysis of variance followed by Student's $t$-test (mitochondrial membrane depolarization and ATP levels). All the values from five replicates are presented as the mean \pm standard error. Treatments were considered statistically different from one another at $\mathrm{p}<0.05$.

\section{RESULTS}

Table 1 shows the effects of 5-ALA on sperm motility parameters during $2 \mathrm{~h}$ of incubation period (Experiment 1 ).

Supplementation with 0.01 and $0.05 \mathrm{mM}$ 5-ALA significantly increased the sperm motility compared to the control group. However, the value of the motility in $0.1 \mathrm{mM} 5$-ALA treatment was similar to the control during the incubation period. Treatment with $0.05 \mathrm{mM}$ 5-ALA showed the highest motility among all treatments.

The sperm progressive motility was significantly increased after 1 and $1.5 \mathrm{~h}$ of incubation. When compared to the control group, addition of 0.01 and $0.05 \mathrm{mM}$ 5-ALA significantly increased the sperm progressive motility; moreover, the 0.05 mM 5-ALA treatment showed the highest value among all treatments. However, treatment with $0.1 \mathrm{mM} 5$-ALA did not improve the sperm progress motility.
The values of sperm LIN were improved after 1, 1.5, and 2 $\mathrm{h}$ of incubation. Treatment with 0.01 and $0.05 \mathrm{mM}$ 5-ALA significantly improved the LIN compared to the control, whereas the $0.1 \mathrm{mM} 5$-ALA treatment did not significantly change compared to the control.

Sperm VAP increased during 1 to $2 \mathrm{~h}$ of incubation, and treatment with $0.05 \mathrm{mM}$ 5-ALA significantly increased the VAP compared to the control at $0.5,1,1.5$, and $2 \mathrm{~h}$ of incubation. The other treatments did not increase sperm VAP during the incubation period.

Treatment with $0.01 \mathrm{mM}$ 5-ALA increased sperm VCL at 1 and $1.5 \mathrm{~h}$ of incubation, but no effect was observed at $2 \mathrm{~h}$ of incubation. Interestingly, compared to the control, treatment with $0.05 \mathrm{mM}$ 5-ALA significantly increased sperm VCL at each point of incubation; however, the $0.1 \mathrm{mM} 5$-ALA treatment did not show any positive effect on the VCL parameter.

Regarding sperm VSL, the value of VSL was significantly increased at 1 and $1.5 \mathrm{~h}$ points of incubation. Treatment with $0.05 \mathrm{mM}$ 5-ALA significantly increased the sperm VSL, whereas treatments with 0.01 and $0.1 \mathrm{mM} \mathrm{5-ALA} \mathrm{did} \mathrm{not}$ improve sperm VSL.

As compared to the control, treatment with $0.01 \mathrm{mM}$ 5 -ALA improved the values of sperm WOB at $1 \mathrm{~h}$ of incubation, but no changes were observed at $1.5 \mathrm{~h}$ and $2 \mathrm{~h}$ of incubation. Interestingly, the sperm WOB was significantly increased when treated with $0.05 \mathrm{mM} 5$-ALA for $2 \mathrm{~h}$. However, the sperm WOB was not increased in the $0.1 \mathrm{mM}$ ALA treatment.

As described above, sperm motility, progressive motility, LIN, VAP, VCL, VSL, and WOB were significantly increased by the $0.05 \mathrm{mM} 5$-ALA treatment after $1.5 \mathrm{~h}$ of incubation (Experiment 1); we also investigated whether the chicken sperm mitochondrial membrane depolarization was altered by 5-ALA. It was observed that addition of 5-ALA significantly increased the MFI of sperm when sperm were analyzed by flow cytometry (Figure 1A-B), which means that the sperm mitochondrial membrane depolarization was increased in the $0.05 \mathrm{mM}$ ALA treatment as the high MFI indicates sperm with high mitochondrial membrane depolarization (Experiment 2). Interestingly, when we measured the sperm ATP level after ALA treatment (supplementation of $0.05 \mathrm{mM}$ 5-ALA and $1.5 \mathrm{~h}$ incubation) with a ATP analyzed kit, it was observed that the ATP level in 5-ALA treatment was significantly $(\mathrm{p}<0.01)$ lower than the control (Figure 2) (Experiment 2).

It was observed that PBS $(+)+$ caffeine treatment significantly decreased sperm motility compared to the PBS (-) treatment, meanwhile, the PBS (+) + 5-ALA increased the motility during the incubation. Moreover, the positive effect of 5-ALA on sperm motility disappeared with the addition of the 5-ALA to the PBS $(+)+$ caffeine treatment, which in- 
Table 1. Effects of various concentrations of 5-aminolevulinic acid and the incubation time for sperm motility parameters

\begin{tabular}{|c|c|c|c|c|c|c|c|c|c|}
\hline \multirow{3}{*}{$\begin{array}{l}\text { Item } \\
\text { Motility }^{2)}(\%)\end{array}$} & \multirow{2}{*}{ Con (mM) } & \multirow{2}{*}{\multicolumn{4}{|c|}{ Time (h) }} & \multirow{3}{*}{ SEM } & \multicolumn{3}{|c|}{ p-vale } \\
\hline & & & & & & & \multirow[t]{2}{*}{ Con $^{1)}$} & \multirow[t]{2}{*}{ Time } & \multirow[t]{2}{*}{ Conxtime } \\
\hline & & $0.5^{\mathrm{A}}$ & $1^{\mathrm{A}}$ & $1.5^{\mathrm{A}}$ & $2^{A}$ & & & & \\
\hline & $0.01^{\mathrm{bc}}$ & 64.9 & 69.2 & 66.8 & 66.6 & & & & \\
\hline & $0.05^{c}$ & 65.1 & 70.7 & 70.6 & 69.0 & & & & \\
\hline & $0.1^{\mathrm{ab}}$ & 63.9 & 65.6 & 62.8 & 61.2 & & & & \\
\hline & $0^{a}$ & 45.6 & 54.5 & 55.2 & 45.7 & 8.1 & $<0.0001$ & 0.0068 & 0.8730 \\
\hline & $0.01^{b c}$ & 52.6 & 59.9 & 57.4 & 56.1 & & & & \\
\hline & $0.05^{c}$ & 54.6 & 61.4 & 63.0 & 59.3 & & & & \\
\hline & $0.1^{\mathrm{ab}}$ & 50.3 & 53.1 & 51.8 & 50.1 & & & & \\
\hline & $0.1^{\mathrm{ab}}$ & 53.2 & 57.4 & 52.5 & 53.4 & & & & \\
\hline \multirow[t]{5}{*}{$\operatorname{VAP}(\mathrm{mm})$} & & $0.5^{\mathrm{A}}$ & $1^{B}$ & $1.5^{\mathrm{B}}$ & $2^{B}$ & & & & \\
\hline & $0^{a}$ & 74.6 & 83.3 & 87.6 & 84.4 & 4.5 & 0.0166 & 0.0001 & 0.9969 \\
\hline & $0.01^{\mathrm{ab}}$ & 75.6 & 88.5 & 91.1 & 82.7 & & & & \\
\hline & $0.05^{b}$ & 83.0 & 94.5 & 97.1 & 90.9 & & & & \\
\hline & $0.1^{\mathrm{ab}}$ & 75.2 & 89.0 & 86.6 & 84.0 & & & & \\
\hline \multirow[t]{3}{*}{ VCL (mm) } & & $0.5^{\mathrm{A}}$ & $1^{\mathrm{B}}$ & $1.5^{\mathrm{B}}$ & $2^{\mathrm{AB}}$ & & & & \\
\hline & $0^{a}$ & 107.1 & 120.7 & 124.7 & 116.5 & 8.2 & 0.0063 & 0.0037 & 0.9914 \\
\hline & $0.01^{\mathrm{ab}}$ & 115.6 & 126.3 & 123.7 & 114.8 & & & & \\
\hline & $0.1^{\mathrm{a}}$ & 95.3 & 109.8 & 105.8 & 101.0 & & & & \\
\hline \multirow[t]{5}{*}{ WOB (\%) } & & $0.5^{\mathrm{A}}$ & $1^{\mathrm{B}}$ & $1.5^{\mathrm{AB}}$ & $2^{A B}$ & & & & \\
\hline & $0^{a}$ & 65.4 & 77.4 & 78.7 & 73.5 & 7.5 & 0.0009 & 0.0191 & 0.5568 \\
\hline & $0.01^{\mathrm{ab}}$ & 74.7 & 82.0 & 80.6 & 73.3 & & & & \\
\hline & $0.05^{b}$ & 76.3 & 86.5 & 83.4 & 81.1 & & & & \\
\hline & $0.1^{\mathrm{a}}$ & 73.5 & 75.0 & 68.1 & 68.8 & & & & \\
\hline
\end{tabular}

Values are least square means.

The data obtained from five replicates were analyzed by two way ANOVA, and compared using Tukey-Kramer's HDS test.

SEM, standard error of the mean; LIN, linearity; VAP, average path velocity; VCL, curvilinear velocity; VSL, straight-line velocity; WOB, wobble.

1) Con., concentration (mM).

2) Motility, percentage of motile sperm moving with path velocity $>12 \mu \mathrm{m} / \mathrm{s}$.

3) Prog. M., percentage of motile sperm moving with path velocity $45 \mu \mathrm{m} / \mathrm{s}$ and in a straight line over $80 \%$ of the time.

${ }^{a-c}$ Different letters within the same column are significantly different $(p<0.05)$

$A, B$ Different letters within the same low are significantly different $(p<0.05)$.

dicated that the 5-ALA could not rescue sperm motility in the treatment of PBS $(+)+$ caffeine (Figure 3A). When we checked sperm progressive motility, the results were similar to those in motility pattern, PBS $(+)+5$-ALA treatment showed the highest progressive motility among all treatments, and
PBS $(+)+$ caffeine decreased the sperm progressive motility. Addition of 5-ALA to the PBS $(+)+$ caffeine treatment could not recover the sperm progressive motility (Figure 3B) (Experiment 3).

As showed in Figure 4A, glucose medium decreased sperm 
A

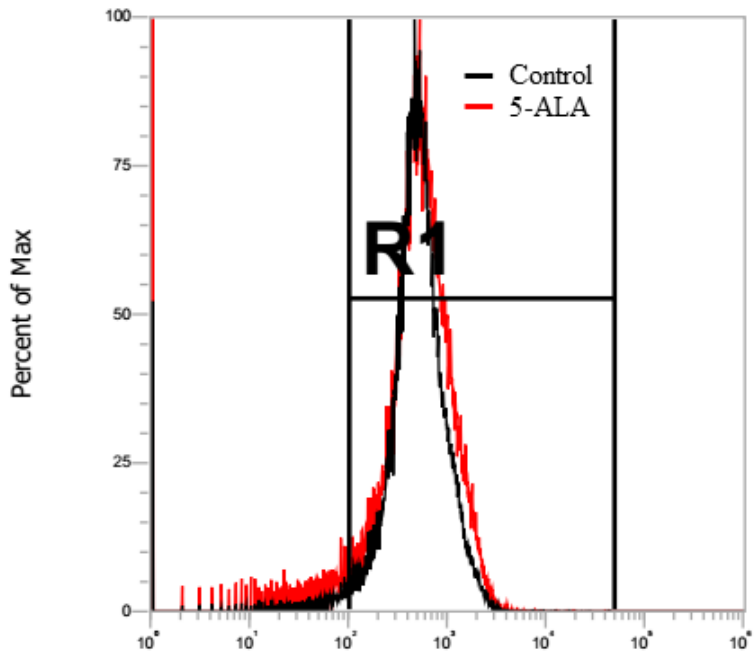

$\mathrm{B}$

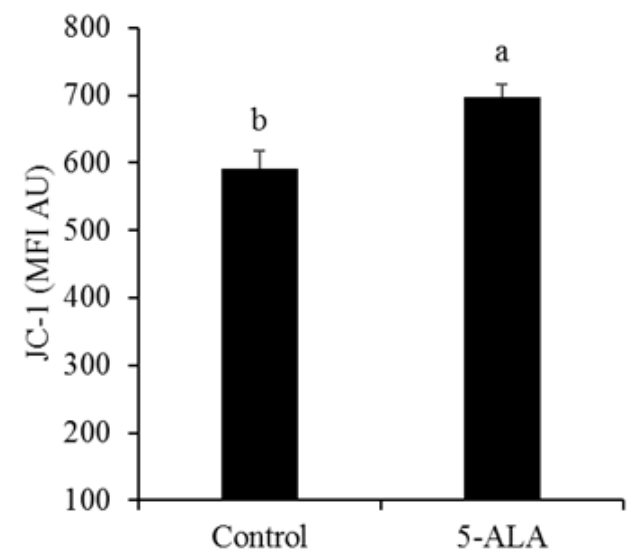

Figure 1. Effect of 5-aminolevulinic acid (5-ALA) treatment on sperm mitochondrial membrane depolarization. The sperm mitochondrial membrane depolarization after $1.5 \mathrm{~h}$ of incubation with $0.05 \mathrm{mM}$ 5-ALA were analyzed with the MitoPT JC-1 Assay kit. (A) Fluorescence intensity of sperm when sperm analyzed by flow cytometry. Gate R1 means the chicken sperm positive stained with JC-1 probe. (B) Mean fluorescence intensity of sperm. Data were obtained from five replicates. ${ }^{a, b}$ Different letters show significant difference $(p<0.05)$.

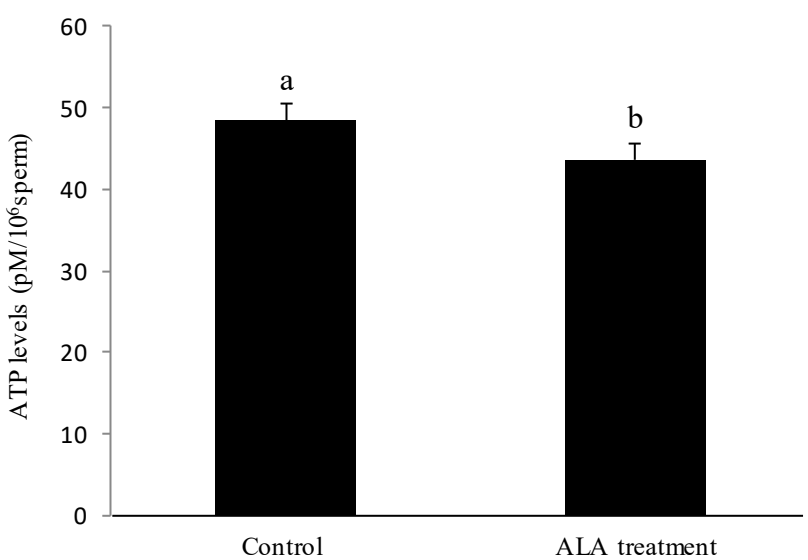

Figure 2. Effect of 5-aminolevulinic acid (5-ALA) treatment on sperm ATP levels. The sperm ATP levels after $1.5 \mathrm{~h}$ of incubation with 0.05 mM 5-ALA were analyzed with the ATP Assay kit. Data were obtained from five replicates. ${ }^{a, b}$ Different letters show significant difference $(p<0.01)$.

motility compared to the PBS (-) treatment. Addition of 5-ALA to the PBS (-) medium significantly increased sperm motility, however, 5-ALA could not increase the motility in the glucose medium. Furthermore, the positive effect of 5-ALA on sperm progressive motility was observed in PBS (-) medium, but it did not increase sperm progressive motility in glucose medium (Figure 4B). Those data indicated that 5-ALA improved chicken motility patterns not rely on enhancing sperm ATP production via glycolysis metabolism (Experiment 4).

\section{DISCUSSION}

To the best of our knowledge, this is the first time the effects of 5-ALA were evaluated in the animal reproduction field. It was observed that treatment with 5-ALA significantly increased chicken sperm motility, progressive motility, LIN, VAP, VCL, VSL, and WOB during the $2 \mathrm{~h}$ of incubation period in present study. Moreover, the sperm treated with $0.05 \mathrm{mM}$ 5-ALA exhibited a high mitochondrial membrane depolarization. These results were consistent with those of previous studies, which reported that the mitochondrial membrane depolarization is highly positively correlated with sperm motility $[20,22,23]$. Mitochondria are one of major energy source generation sites in the sperm, and the sperm with high mitochondrial membrane depolarization indicates that they have high linear motility $[19,20]$. As the chicken sperm mitochondrial membrane depolarization was activated by $0.05 \mathrm{mM} 5$-ALA, the sperm was provided with amounts of energy for continuous movement, thus increasing the sperm motility patterns in this study. These data were similar to those of Chiabrando et al [5] who reported that 5-ALA is an essential substrate for enhancing the oxygen metabolism and energy generation in the animals. Moreover, KlimowiczBodys et al [24] found that sperm motility and progressive motility were also positively correlated with sperm viability in birds when the CASA was used to analyze sperm motility patterns, indicating that the viability of the chicken sperm in the present study was also improved by treatment with 5-ALA. The 5-ALA was beneficial for improving chicken sperm motility patterns during the incubation. 

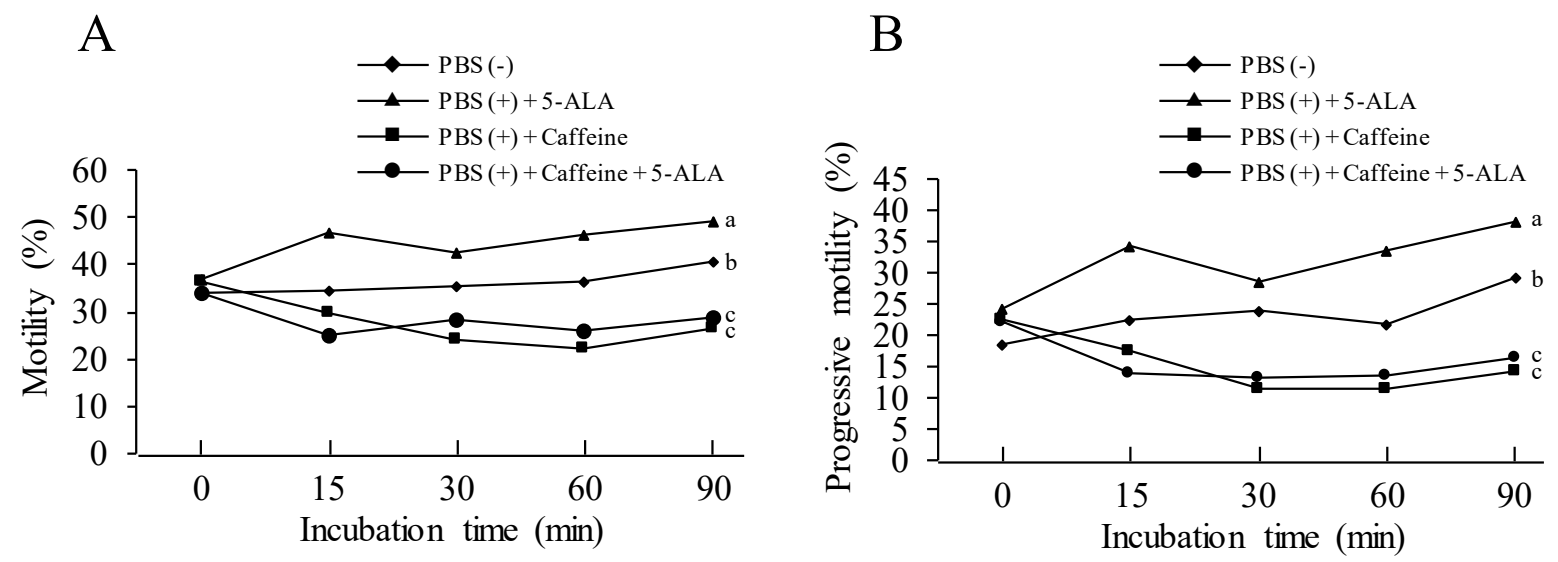

Figure 3. Effects of 5-aminolevulinic acid (5-ALA), caffeine and $\mathrm{Ca}^{2+}$ on sperm motility. The washed sperm was suspended with mediums of phosphate-buffered saline (PBS (-)), PBS (+) + 5-ALA, PBS $(+)+$ caffeine and PBS $(+)+$ caffeine +5 -ALA. The sperm motility was evaluated at 15,30 , 60 , and 90 min points of incubation by computer-assisted sperm analysis. Data were obtained from five replicates. ${ }^{\text {a-c }}$ Different letters show significant difference $(p<0.05)$.
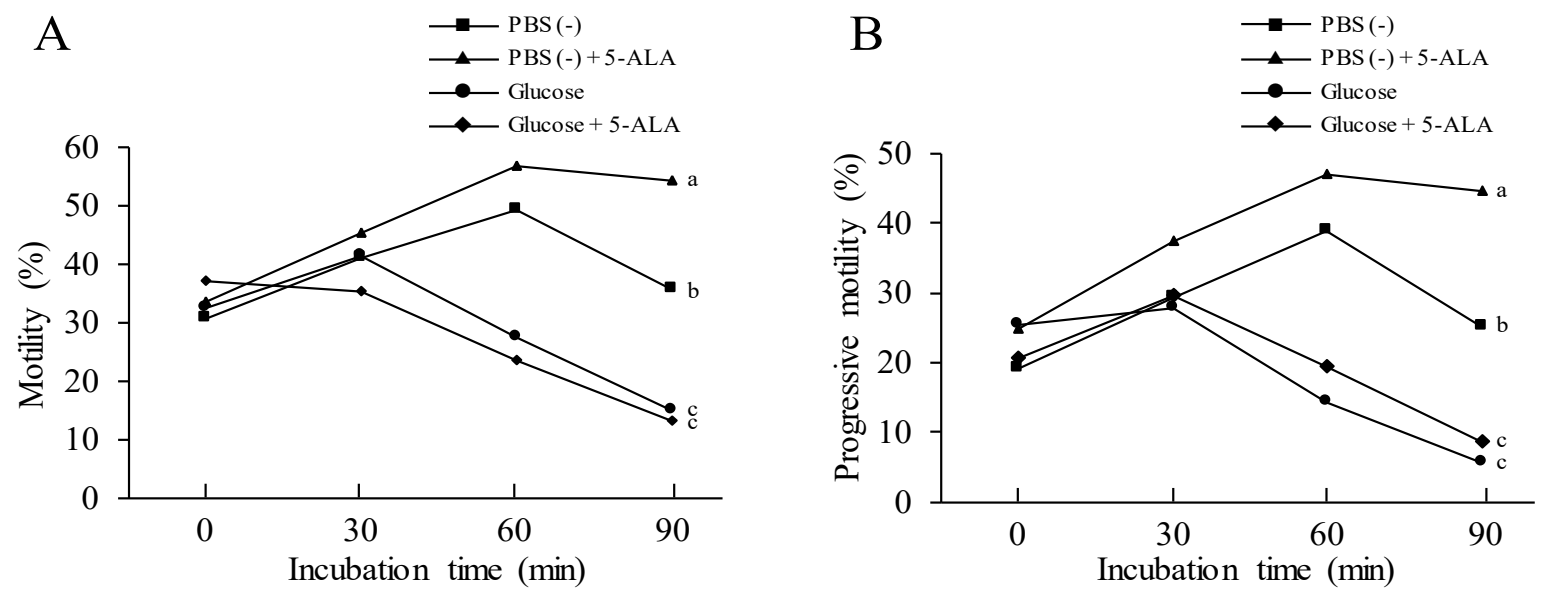

Figure 4. Effects of 5-aminolevulinic acid (5-ALA) and glucose on sperm motility. The washed sperm was incubated with mediums of phosphate-buffered saline (PBS (-)), PBS (-) + 5-ALA, glucose, glucose + 5-ALA for $1.5 \mathrm{~h}$. The sperm motility was evaluated every 30 min by computer-assisted sperm analysis. Data were obtained from five replicates. ${ }^{a-c}$ Different letters show significant difference $(p<0.05)$.

Chicken sperm mitochondrial membrane depolarization was enhanced by the 5-ALA treatment in the present study, which was consistent with the previous studies that the 5-ALA could stimulate the mitochondrial function in somatic cells $[25,26]$. Wachowska et al [27] demonstrated that the 5-ALA is an endogenous amino acid and an intermediate involved in heme biosynthesis in mitochondria. There are two steps involved in heme synthesis from 5-ALA: firstly, the endogenous or the exogenous 5-ALA is metabolized as a substrate for protoporphyrin IX, then the cell inserts a ferrous ion into protoporphyrin IX to generate heme. Moreover, heme acts as a protein-bound prosthetic group in the mitochondrial respiratory chain complexes II, III, and IV and cytochrome C [28], thus, the activity of the respiratory chain complexes is depended on the heme level, which indicates that the mi- tochondrial function is regulated by the heme. In present study, addition of 5-ALA significantly increased the sperm mitochondrial membrane depolarization (Figure 1A-B) maybe because the sperm utilizes 5-ALA to synthesise heme. It was supported by Shimura et al [29] who observed that 5-ALA upregulated oxidative phosphorylation proteins with increased oxidative phosphorylation gene expression in human skin fibroblasts. The result also agreed with the observation that 5-ALA was necessary to produce heme proteins [26,30-32]. Therefore, sperm may utilize the exogenous 5-ALA to produce heme to enhance mitochondrial function.

Sperm ATP is generated by the glycolysis and mitochondrial oxidative phosphorylation pathways [33]. In present study, it was observed that the ATP levels in the sperm were decreased by addition of $0.05 \mathrm{mM}$ ALA to the diluted medi- 
um after $1.5 \mathrm{~h}$ of incubation. The results showed that the sperm ATP levels are negatively correlated with the data on linear motility patterns, which might be because the total levels of ATP rather than the mitochondria-generated ATP levels were measured in this study. Zhu et al [20] reported that only the ATPs generated from the mitochondrial oxidative phosphorylation are positively correlated with linear motility of the spermatozoa. Furthermore, when we added 5-ALA to the glucose medium, the sperm motility patterns were not increased (Figure 4A-B), which indicated that the 5-ALA could not enhance ATP production in sperm. Chicken sperm may utilize the 5-ALA for stimulating ATP utilization rather than ATP generation to increase sperm motility in this study. As sperm motility is correlated with both the ability to produce sufficient ATP and the ability to utilize ATP effectively [34], the ATP level in sperm will be decreased under the condition that sperm utilize the ATP for flagellum movement without enhancement of ATP generation. This supports our result in which a reduction of ATP levels was observed in the 5-ALA treatment in present study. Unfortunately, the mechanism of how 5-ALA stimulates chicken sperm utilization of ATP is not clear, and needs further study.

Regarding the regulation of the chicken sperm motility, Wishart and Ashizawa [16] reported that calcium and cAMP were the main regulators of sperm motility, and caffeine, but not calcium, caused an increase in sperm cAMP levels. In this study, the caffeine treatment could not recover the sperm motility (Figure 3A-B). Therefore, it is considered that 5-ALA and caffeine might be different functions to increase chicken sperm motility.

It was observed that the $0.05 \mathrm{mM}$ is the best concentration of 5-ALA to improve the chicken sperm quality, while the high concentration $(0.1 \mathrm{mM})$ had a negative effect on sperm motility in this study. The results were similar to the ones reported by Shimamura et al [35] who reported that high concentrations of 5-ALA resulted in a decrease in the cell viability. Previous studies showed that 5-ALA increased the reactive oxygen species (ROS) in the cells $[35,36]$; therefore, high level of 5-ALA might increase the chicken sperm ROS levels, and thus decreasing the sperm linear motility compared to the $0.05 \mathrm{mM}$ 5-ALA treatment.

In conclusion, the present study is the first report on the effect of 5-ALA on sperm quality. We found that the supplementation with $0.05 \mathrm{mM}$ 5-ALA significantly increased the chicken sperm motility, progressive motility, LIN, VAP, VCL, VSL, and the WOB. Moreover, sperm mitochondrial membrane depolarization was also increased by treatment with 0.05 mM 5-ALA. Interestingly, addition of 0.05 mM 5-ALA to the PBS medium decreased the sperm ATP levels after 1.5 $h$ of incubation, whereas the sperm motility could not be increased by $0.05 \mathrm{mM} 5$-ALA treatment in the glucose medium. Therefore, 5-ALA might increase sperm mitochondrial mem- brane depolarization to utilize the ATP for enhancing sperm movement.

\section{CONFLICT OF INTEREST}

We certify that there is no conflict of interest with any financial organization regarding the material discussed in the manuscript. Taniguchi S is an employee of Neopharma Japan Co., Ltd..

\section{REFERENCES}

1. Morton AK, Kushner PJ, Straka GJ, Burnham FB. Biosynthesis of 5-aminolevulinic acid and heme from 4,5-dioxovalerate in the rat. J Clin Inve 1983;71:1744-9. https://doi.org/10.1172/ JCI110929

2. Korkmaz A. Effects of exogenous application of 5-aminolevulinic acid in crop plants. In: Ahmad P, Prasad MNV, editors. Abiotic stress responses in plants. New York, NY, USA: Springer; 2012. https://doi.org/10.1007/978-1-4614-0634$1 \_12$

3. Liu L, Nguyen NT, Ueda A, Saneoka H. Effects of 5-aminolevulinic acid on Swiss chard (Beta vulgaris L. subsp. cicla) seedling growth under saline conditions. Plant Growth Regul 2014;74:219-28. https://doi.org/10.1007/s10725-014-9913-0

4. Kang Z, Ding W, Gong X, Liu Q, Du G, Chen J. Recent advances in production of 5-aminolevulinic acid using biological strategies. World J Microbiol Biotechnol 2017;33:200. https://doi.org/10.1007/s11274-017-2366-7

5. Chiabrando D, Vinchi F, Fiorito V, Mercurio S, Tolosano E. Heme in pathophysiology: a matter of scavenging, metabolism and trafficking across cell membranes. Front Pharmacol 2014; 5:61. https://doi.org/10.3389/fphar.2014.00061

6. Morokuma Y, Yamazaki M, Maeda T, et al. Hair growth stimulatory effect by a combination of 5-aminolevulinic acid and iron ion. Int J Dermatol 2008;47:1298-303. https:// doi.org/10.1111/j.1365-4632.2008.03783.x

7. Lee WJ, Sim HB, Jang YH, Lee S-J, Kim DW, Yim S-H. Efficacy of a complex of 5-aminolevulinic acid and glycylhistidyl-lysine peptide on hair growth. Ann Dermatol 2016; 28:438-43. https://doi.org/10.5021/ad.2016.28.4.438

8. Sato K, Matsushita K, Takahashi K, et al. Dietary supplementation with 5-aminolevulinic acid modulates growth performance and inflammatory responses in broiler chickens. Poult Sci 2012;91:1582-9. https://doi.org/10.3382/ps.2010-01201

9. Sugiyama Y, Hiraiwa Y, Hagiya Y, Nakajima M, Tanaka T, Ogura S. 5-Aminolevulinic acid regulates the immune response in LPS-stimulated RAW 264.7 macrophages. BMC Immunol 2018;19:41. https://doi.org/10.1186/s12865-0180277-5

10. Marbacher S, Klinger E, Schwyzer L, et al. Use of fluorescence to guide resection or biopsy of primary brain tumors and 
brain metastases. J Neurosurg 2014;36:E10. https://doi.org/ 10.3171/2013.12.FOCUS13464

11. Schatlo B, Fandino J, Smoll NR, et al. Outcomes after combined use of intraoperative MRI and 5-aminolevulinic acid in high-grade glioma surgery. Neuro-Oncology 2015;17:15607. https://doi.org/10.1093/neuonc/nov049

12. Hotta Y, Tanaka T, Takaoka H, Takeuchi Y, Konnai M. Promotive effects of 5-aminolevulinic acid on the yield of several crops. Plant Growth Regul 1997;22:109-14. https://doi.org/10. 1023/A:1005883930727

13. Hara M, Takahashi I, Yamori M, Tanaka T, Funada S, Watanabe K. Effects of 5-aminolevulinic acid on growth and amylase activity in the radish taproot. Plant Growth Regul 2011;64: 287-91. https://doi.org/10.1007/s10725-010-9542-1

14. Watanabe K, Tanaka T, Hotta Y, Kuramochi H, Takeuchi Y. Improving salt tolerance of cotton seedlings with 5-aminolevulinic acid. Plant Growth Regul 2000;32:97-101. https:// doi.org/10.1023/A:1006369404273

15. Hotta Y, Tanaka T, Bingshan L, Takeuchi Y, Konnai M. Improvement of cold resistance in rice seedlings by 5 -aminolevulinic acid. J Pestic Sci 1998;23:29-33. https://doi.org/10. 1584/jpestics.23.29

16. Wishart GJ, Ashizawa K. Regulation of the motility of fowl spermatozoa by calcium and cAMP. J Reprod Fertil 1987;80: 607-11. https://doi.org/10.1530/jrf.0.0800607

17. Burrows WH, Quinn JP. The collection of spermatozoa from the domestic fowl and turkey. Poult Sci 1937;16:19-24. https:// doi.org/10.3382/ps.0160019

18. Maeda T, Terada T, Tsutsumi Y. The efficacy of glucose and electrolyte solutions in preserving the structure of fowl spermatozoa after freezing and thawing. Jpn Poult Sci 1985;22: 201-8. https://doi.org/10.2141/jpsa.22.201

19.Zhu Z, Kawai T, Umehara T, Hoque SAM, Zeng W, Shimada $M$. Negative effects of ROS generated during linear sperm motility on gene expression and ATP generation in boar sperm mitochondria. Free Radical Biol Med 2019;141:15971. https://doi.org/10.1016/j.freeradbiomed.2019.06.018

20.Zhu Z, Umehara T, Okazaki T, et al. Gene expression and protein synthesis in mitochondria enhance the duration of high-speed linear motility in boar sperm. Front Physiol 2019; 10:252. https://doi.org/10.3389/fphys.2019.00252

21.Matsuzaki M, Mizushima S, Ichikawa Y, Shiba K, Inaba K, Sasanami T. Effects of a protein kinase inhibitor on sperm motility in the Japanese quail. J Poult Sci 2017;54:73-9. https:// doi.org/10.2141/jpsa.0160079

22. Garner DL, Thomas CA, Joerg HW, DeJarnette JM, Marshall CE. Fluorometric assessments of mitochondrial function and viability in cryopreserved bovine spermatozoa. Biol Reprod 1997;57:1401-6. https://doi.org/10.1095/biolreprod57.6.1401

23.Zhu Z, Fan X, Lv Y, Lin Y, Wu D, Zeng W. Glutamine protects rabbit spermatozoa against oxidative stress via glutathione synthesis during cryopreservation. Reprod Fertil Dev 2017;
29:2183-94. https://doi.org/10.1071/RD17020

24. Klimowicz-Bodys MD, Batkowski F, Ochrem AS, Savic MA. Comparison of assessment of pigeon sperm viability by contrast-phase microscope (eosin-nigrosin staining) and flow cytometry (SYBR-14/propidium iodide (PI) staining). Theriogenology 2012;77:628-35. https://doi.org/10.1016/j. theriogenology.2011.09.001

25. Ogura S, Maruyama K, Hagiya Y, et al. The effect of 5-aminolevulinic acid on cytochrome $\mathrm{c}$ oxidase activity in mouse liver. BMC Res Notes 2011;4:66. https://doi.org/10.1186/ 1756-0500-4-66

26. Ota U, Hara T, Nakagawa H, et al. 5-Aminolevulinic acid combined with ferrous ion reduces adiposity and improves glucose tolerance in diet-induced obese mice via enhancing mitochondrial function. BMC Pharmacol Toxicol 2017;18:7. https://doi.org/10.1186/s40360-016-0108-3

27. Wachowska M, Muchowicz A, Firczuk M, et al. Aminolevulinic acid (ALA) as a prodrug in photodynamic therapy of cancer. Molecules 2011;16:4140-64. https://doi.org/10.3390/ molecules 16054140

28. Kim HJ, Khalimonchuk O, Smith MP, Winge DR. Structure, function, and assembly of heme centers in mitochondrial respiratory complexes. Biochim Biophys Acta Mol Cell Res 2012;1823:1604-16. https://doi.org/10.1016/j.bbamcr.2012. 04.008

29. Shimura M, Nozawa N, Ogawa-Tominaga M, et al. Effects of 5-aminolevulinic acid and sodium ferrous citrate on fibroblasts from individuals with mitochondrial diseases. Sci Rep 2019;9:10549. https://doi.org/10.1038/s41598-01946772-x

30. Mingone JC, Gupte AS, Chow LJ, Ahmad M, Abraham GN, Wolin SM. Protoporphyrin IX generation from delta-aminolevulinic acid elicits pulmonary artery relaxation and soluble guanylate cyclase activation. Am J Physiol-Lung Cell Mol Physiol 2006;291:337-44. https://doi.org/10.1152/ajplung. 00482.2005

31. Miura M, Ito K, Hayashi M, Nakajima M, Tanaka T, Ogura S. The effect of 5-aminolevulinic acid on cytochrome P450mediated prodrug activation. PloS One 2015;10:e131793. https://doi.org/10.1371/journal.pone.0131793

32.Nishio Y, Fujino M, Zhao M, et al. 5-Aminolevulinic acid combined with ferrous iron enhances the expression of heme oxygenase-1. Int Immunopharmacol 2014;19:300-7. https://doi.org/10.1016/j.intimp.2014.02.003

33. Plessis SS, Agarwal A, Mohanty G, Linde MVD. Oxidative phosphorylation versus glycolysis: what fuel do spermatozoa use? Asian J Androl 2015;17:230-5. https://doi.org/10.4103/ 1008-682X.135123

34. Toit DD, Bornman MS, Van Der Merwe MP, Du Plessis DJ, Oosthuizen JMC. Differential sperm motility scoring and sperm ATP concentrations. Arch Androl 1993;30:69-71. https://doi.org/10.3109/01485019308988371 


\section{$A 3^{\prime}$}

35. Shimamura Y, Tamatani D, Kuniyasu S, et al. 5-Aminolevulinic acid enhances ultrasound-mediated antitumor activity via mitochondrial oxidative damage in breast cancer. Anticancer Res 2016;36:3607-12.

36.Ito H, Tamura M, Matsui H, Majima JH, Indo HP, Hyodo I.
Reactive oxygen species involved cancer cellular specific 5 -aminolevulinic acid uptake in gastric epithelial cells. J Clin Biochem Nutr 2014;54:81-5. https://doi.org/10.3164/ jcbn.13-98 